sichtspunkten erfolgte (S. 40). Wegen ihrer grundlegenden Bedeutung für den Verlauf der französischen Debatte wird mit einem Aufsatz zur Produktzyklustheorie des US-Amerikaners Raymond Vernon begonnen. Es folgen Beiträge von Christian Palloix und Michel Aglietta zur Internationalisierung des Kapitalkreislaufs innerhalb einer Branche und anschließend ein Beitrag zum Konzept der sektoralen Betrachtung (W. Andreff), dessen Bedeutung für die weitere Diskussion in einigen Texten aus einer Studie des französischen Industrieministeriums über die internationale Arbeitsteilung dokumentiert wird.

Ein neuerer Aufsatz von Christian Palloix soll schließlich den Erklärungswert der vorangestellten Ansätze für die gegenwärtigen Veränderungen der internationalen Arbeitsteilung aufzeigen. Er geht davon aus, daß die augenblickliche Krise eine organische Krise des Kapitalismus sei und kommt zu dem Ergebnis, daß sie nur durch die Ausdehnung des Verwertungsprozesses des Kapitals auf die unterentwickelten Gesellschaftsformationen - d. h. durch die Auslagerung von Produktionen in die Billiglohnländer der sogenannten Dritten Welt - (S. 250) gelöst werden kann. Die Textauswahl wird durch eine umfangreiche Bibliographie am Schluß des Bandes ergänzt.

Das Anliegen der Forschungsgruppe, den ,'Gang der Diskussion nicht in allen seinen Verästelungen nachzuzeichnen" (S. 40), sondern jeden Theorieansatz in seiner entwickeltsten Form zu präsentieren, ermöglicht dem Leser, sich anhand der Textauswahl rasch über die wesentlichen Inhalte der Internationalisierungsdebatte zu informieren. Der weitgehende Verzicht auf eine systematische Darstellung und die Präsentation einiger Positionen durch ihre Rezeption in bereits wieder weiterführenden Arbeiten setzen jedoch voraus, daß der Leser geneigt ist, sich auf Einschätzung und Auswahl der Forschungs- und Herausgebergruppe zu verlassen. Dies gilt um so mehr, als die Gruppe darauf verzichtet, bereits in diesem ersten Arbeitsergebnis des Forschungsprojektes ihren theoretischen Standort deutlich auszuweisen (s. Vorwort S. 8). Die Einbeziehung von originären, wenn auch noch nicht ausgereif ten Texten vor dem Hintergrund ihres Entstehungszusammenhangs wäre in einigen Fällen sicher sinnvoll gewesen, zumal auch die als am meisten entwickelte Form des jeweiligen Ansatzes vorgestellten Texte auf viele Fragen, die im Zusammenhang mit dem Internationalisierungsprozeß entstehen, keine Antwort geben. So weist die Gruppe zum Schluß selbst darauf hin, daß die Verstärkung der regulativen Funktionen des Staates im Verlauf der ökonomischen Verflechtung nicht angemessen berücksichtigt werde. Ferner erscheint die Annahme einer festen Hierarchie der internationalen Arbeitsteilung fraglich. Die wachsende Bedeutung der Dritten Welt für die erweiterte Reproduktion in den kapitalistischen Industriestaaten unter der Bedingung zunehmender innerkapitalistischer Konkurrenz wird ebenfalls nicht hinreichend erfaßt. Die Beantwortung dieser Fragen bleibt damit der weiteren wissenschaftlichen Diskussion und Forschung vorbehalten. Im Hinblick darauf ist anzunehmen, daß die Forschungsgruppe ihr Ziel, mit diesem Reader den Diskussionsprozeß in der Bundesrepublik anzuregen, erreichen wird.

Hella Gerth-Wellmann

\title{
Alfred Schmidt
}

Internationale Arbeitsteilung oder ungleicher Tausch: Kontroversen über den Handel zwischen Industrie- und Entwicklungsländern

Frankfurt/M., New York, Campus Verlag, 1978, 269 S., 34,- DM

Seit Ricardo sein Theorem der komparativen Kostenvorteile aufstellte, wird in den meisten Arbeiten zur realen Außenhandelstheorie zunächst einmal davon ausgegangen, daß die Aufnahme von Handelsbeziehungen für alle beteiligten Länder nur Vorteile bringen könne. 
Zwar kann die Verteilung der Handelsgewinne ungleich sein; eine absolute Verschlechterung des Wohlstandes eines Landes ist bei Ricardo jedoch nicht möglich.

Die praktische Anwendbarkeit des Theorems für die Handelspolitik ist natürlich nicht unbestritten geblieben. Besonders in bezug auf den Handel zwischen Industrie- und Entwicklungsländern, zwischen Metropolen und Peripherien gibt es zahlreiche Stimmen, die hier Freihandel nicht für angebracht halten und schädliche Auswirkungen eines ungezügelten Außenhandels auf die Entwicklungsmöglichkeiten der Dritten Welt zu sehen glauben. Aber selbst die meisten Gegner des Freihandels akzeptieren die statischen Vorteile, die eine Spezialisierung à la Ricardo verspricht. Sie behaupten lediglich, durch eine derartige, den Marktkräften überlassene Integration der Dritten Welt in die Weltwirtschaft werde eine notwendige und mögliche Veränderung der komparativen Vorteile und der Spezialisierungsmuster im Zeitablauf verhindert. Das Erziehungszollargument von Friedrich List und die These von Prebish/Singer/Myrdal über die säkulare Verschlechterung der terms of trade für Entwicklungsländer als Lieferanten von Primärgütern lassen die Möglichkeit statischer Handelsgewinne durchaus gelten. Nur im Zeitablauf ergeben sich negative Auswirkungen. In gleicher Weise bestätigt zum Beispiel auch Senghaas die statischen Vorteile des internationalen Handels für Entwicklungsländer. Er postuliert jedoch eine Entwicklung der produktiven Kräfte, die nur durch die Lernkosten, die bei einem Verzicht auf die statischen Vorteile des Handels entstehen, erreicht werden könne.

Stimmen, die schon die statische Vorteilhaftigkeit von Außenhandel in Frage stellen, sind demgegenüber seltener. Sie behaupten entweder, daß Ricardos Konkurrenzannahme unrealistisch sei - so in der Imperialismustheorie -, oder sie behandeln die negativen Konsequenzen von verzerrten Produktpreisen, die in Entwicklungsländern häufiger anzutreffen sind als in den Industrieländern, für deren Wohlfahrt.

Auch die Thesen vom ungleichen Tausch bestreiten die statische allseitige Vorteilhaftigkeit des Freihandels, beanspruchen aber darüber hinaus, generell für den Handel zwischen Industrie- und Entwicklungsländern Gültigkeit zu haben. Ihre Vertreter bemühen sich, internationale Ausbeutung der Entwicklungsländer durch Außenhandel auch unter Konkurrenzbedingungen nachzuweisen. Durch den Handel würden den ärmeren Ländern Ressourcen entzogen und in die Industrieländer transferiert. Außenhandel wäre damit, im Gegensatz zu Ricardos Theorem, schon in statischer Betrachtungsweise als eine Ursache der Unterentwicklung anzusehen.

Was ist ungleicher Tausch? Schmidt behandelt in seinem Buch die Modelle der zwei wichtigsten Vertreter, Gunther Kohlmey und Arghiri Emmanuel. Für beide ist ungleicher Tausch ein Austausch von Gütern zu einem Preisverhältnis, bei dem nicht eine Einheit lebendiger menschlicher Arbeit eines Landes gegen eine entsprechende Einheit des anderen Landes ausgetauscht wird. In marxistischer Terminologie ist ungleicher Tausch ein nicht-äquivalenter Tausch.

Das Modell von Kohlmey entspricht in seinen Voraussetzungen dem von Ricardo. Es werden zwei Länder betrachtet, in denen unterschiedliche Produktionstechniken angewendet werden: ein Land soll technisch dem anderen in allen Bereichen unterlegen sein. Sowohl Arbeit als auch Kapital seien national mobil, international jedoch immobil. Unter diesen Bedingungen wird das Land mit der weniger effizienten Technik immer mehr Arbeit für seine Exportprodukte aufwenden müssen, als in dem anderen Land für die Herstellung der dafür getauschten Güter notwendig war. Da der Tausch kein Äquivalententausch ist, ist er ungleich. Er wäre allerdings auch dann noch ungleich, wenn dem Land mit der weniger effizienten Technik der gesamte Handelsgewinn zufallen würde. Ein ,,gleicher“ Tausch ist auf 
freiwilliger Basis unter den Bedingungen Kohlmeys nicht denkbar. Der behauptete Werttransfer ist damit rein hypothetischer Natur.

Im Gegensatz zu Kohlmey greift Emmanuel nicht direkt auf das Modell von Ricardo zurück, sondern nimmt vielmehr an, daß lediglich die Arbeitskräfte international immobil seien. Internationale Kapitalbewegungen läßt er demgegenüber ausdrücklich zu. Die daraus resultierende Angleichung der Profitraten in beiden Ländern führt bei ihm zum ungleichen Tausch und dem Transfer von Werten von einem Land in das andere.

Schmidt diskutiert die Thesen beider Autoren, indem er sie, ebenso wie vorher das Modell Ricardos und die Einwände von als bürgerlich einzustufenden Ökonomen (unter anderen Samuelson), in der Form eines linearen Produktionsmodells darstellt. So ist er in der Lage, auch die implizit gemachten Annahmen herauszuarbeiten und modellimmanente Widersprüche aufzuzeigen. Entsprechend dem wirtschaftstheoretischen Charakter seiner Arbeit ist diese Darstellung sehr formal, mit mathematischen Ausdrücken gespickt, schwer zu lesen und für den, der keine gründlichen Vorkenntnisse in lineraren Produktionsmodellen hat, kaum nachvollziehbar.

Der Leser wird jedoch teilweise durch eine ausführliche verbale Zusammenfassung der Ergebnisse im 4. Kapitel entschädigt. Schmidts darin geäußerte Kritik an den Thesen vom ungleichen Tausch ist nicht weniger als vernichtend zu nennen. Er kommt zu dem Ergebnis, daß sich die verwendeten Instrumentarien nicht zur Untersuchung von statischen Handelssituationen eignen. Wenn ferner ein Werttransfer in der von Kohlmey und Emmanuel unterstellten Form stattfände, müßten Bedingungen vorliegen, die mit den Freihandels- und Konkurrenzannahmen unvereinbar sind. Von diesen Annahmen gehen beide Autoren jedoch aus. Ihre Theorien sind daher in sich widersprüchlich. Nach Schmidt vergleichen beide lediglich das Austauschverhältnis, das unter kapitalistischen Bedingungen zustande kommt, mit einem als wünschenswert angesehenen hypothetischen Preisverhältnis. ,Ein solcher Vergleich eignet sich aber nicht für eine positive Erklärung der Auswirkungen des internationalen Handels auf die Produktions- und Konsummöglichkeiten. “Und weiter: ,,Die Thesen vom ungleichen Tausch laufen in sämtlichen hier behandelten Versionen auf die Feststellung hinaus, daß die faktoralen Austauschverhältnisse (das Austauschverhältnis der Arbeitsstunden, die für Exporte und Importe benötigt werden, D. O.) für das Land mit ineffizienter Produktionstechnik und niedrigem Lohnsatz günstiger wären, wenn weltweit die gleiche Produktionstechnik angewandt würde und die internen Verteilungsparameter überall gleich wären. Die Binsenweisheit, die ein solcher hypothetischer Vergleich zutage fördert, bedarf offensichtlich keiner theoretischen Begründung." (S. 229.) Die Thesen vom ungleichen Tausch liefen auf die Forderung hinaus, daß gleiche Arbeit mit gleichem Lohn bedacht werden müsse.

Schmidt sieht also in den Thesen vom ungleichen Tausch keinen Grund dafür, daß sich Außenhandel zuungunsten der Entwicklungsländer auswirken soll und sie besser beraten wären, auf jeglichen Außenhandel zu verzichten. Dies soll nun nicht heißen, daß eine exportorientierte Entwicklungsstrategie einer binnenmarktorientierten vorzuziehen ist. Auch die bürgerlichen Autoren behandeln durchaus Fälle, in denen Außenhandel zu einem Ressourcentransfer führen kann, der ein Land ärmer macht als im Autarkiezustand. Aber, so resümiert Schmid t: ,,Bei beiden Vertretern der These kann nicht die Rede davon sein, daß sie eine fruchtbare Weiterentwicklung der klassischen Außenhandelstheorie vorlegen. Vielmehr fallen sie mit ihren Analyseinstrumenten hinter den Erkenntnisstand der Klassiker zurück." (S. 232) . . , ,Die Thesen vom ungleichen Tausch lassen aufgrund des Vorverständnisses und der Fragestellung, mit der ihre Vertreter die Analyse internationaler Wirtschaftsbezie- 
hungen angehen, keine Ergebnisse erwarten, die nicht - theoretisch besser fundiert - auch im Rahmen wirtschaftstheoretischer Ansätze gewonnen werden können." (S. 237.)

Es ist das besondere Verdienst Schmidts, bürgerliche und marxistische Theorieansätze miteinander zu konfrontieren. Nur schade, daß eine doch so grundlegende Fragestellung nicht allgemeinverständlicher und leserfreundlicher behandelt worden ist.

Dieter Orlowski

BASSAM TIBI

Internationale Politik und Entwicklungsländerforschung.

Materialien zu einer ideologiekritischen Entwicklungssoziologie.

Frankfurt/M. 1979, Edition Suhrkamp, Bd. 983, 225 S., DM 10,--

Dieser Materialienband enthält zehn bereits in diversen Fachzeitschriften in den Jahren 1969 und 1974 publizierte, jedoch überarbeitete und in ihrer Annotation aktualisierte Aufsätze/Rezensionen sowie zwei Originalbeiträge: 1. Tom Mboya (1930-1969). Der Werdegang eines afrikanischen Nationalisten und 2. Tunesien in der Entwicklungsländerforschung der Bundesrepublik Deutschland.

In der Tradition der Kritischen Theorie der Frankfurter Schule stehend, begreift Bassam Tibi die sozialwissenschaftliche Disziplin der Internationalen Politik als eine emanzipatorische Wissenschaft. Mit diesem emanzipatorischen Anspruch intendiert der Autor aus seiner Kritik an der gängigen Entwicklungsländerforschung (i. S. von bürgerlicher Sozialwissenschaft) „Ansätze zu einer kritischen Entwicklungssoziologie aufzuspüren“ (S. 8) und ,,eine alternative Forschungsstrategie zu entfalten" (S. 17).

Seine Forschungsarbeiten haben eine doppelte, aber untrennbar miteinander verknüpfte Zielrichtung: zum einen eine ideologiekritische Auseinandersetzung mit herrschenden Theorieansätzen der Entwicklungsforschung und zum anderen eine ,, handfeste“ Kritik an einer Wissenschaft zu leisten, die Herrschaftswissen produziert, das zum Krisenmanagement und speziell zur Unterdrückung und Ausbeutung der Massen in der Dritten Welt utilisiert und dabei gleichzeitig zu ihrer Legitimation funktionalisiert wird. Als literarische Illustration dient B. Tibi das Brechtsche Zitat des ,Galilei“, der Wissenschaftler verächtlich als „,ein Geschlecht erfinderischer Zwerge (charakterisiert), die für alles gemietet werden können".

Als beispielhaft für diese Wissenschaftsrichtung diskutiert B. Tibi Arbeiten des Freiburger Arnold-Bergsträsser-Instituts und die Entwicklungssoziologie von W. L. Bühl, deren system-theoretischer Ansatz sie letztlich als ,,extreme Ethnozentristen“ ausweist. Ähnlich lautet die Kritik an R. F. Behrendts Reziprozität unterstellendem Akkulturationsbegriff, der jedoch angesichts des asymmetrischen Interaktionsverhältnisses des Kolonialismus vielmehr als ein ,,legitimationsideologisches Instrument“ zur Verschleierung von Ausbeutung und Abhängigkeit fungiert.

Während Tibi der metropolitanen Forschung Ahistorizismus und fehlende Verknüpfung mit den jeweiligen sozio-ökonomischen Strukturen vorwirft, kritisiert er mit gleicher Vehemenz die in der Dritten Welt entwickelten Theorien als ,,romantizistischen Antiindustrialismus", der gerade auch bei einer Symbolfigur wie Gandhi besonders ausgeprägt ist. Die Rigidität des Antiindustrialismus ist sicher zu verurteilen, aber es scheint angesichts der dominant agrarischen Strukturen der Peripherieländer und der Notwendigkeit einer umfassenden Agrarentwicklungsstrategie nicht gerechtfertigt, den Antiindustrialismus als ,,Denkweise einer agrarischen Gesellschaft" (S. 30), die präkoloniale Dorfstrukturen verherrlicht, schlicht als Romantizismus zu reduzieren und ihm jegliche progressive soziale Funktion von vornherein abzusprechen. 